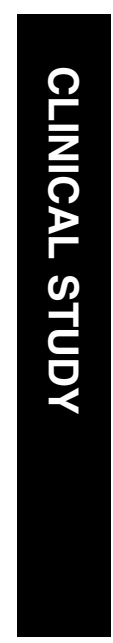

\section{Temporary cosmetic painted prostheses in anophthalmic surgery: an alternative to early postoperative clear conformers}

${ }^{1}$ Corneo-Plastic Unit, Queen Victoria Hospital, East Grinstead, UK

${ }^{2}$ Department of Maxillofacial Prosthetics, Queen Victoria Hospital, East Grinstead, UK

${ }^{3}$ Department of Ophthalmology, Birmingham Children's Hospital, Birmingham, UK

${ }^{4}$ National Artificial Eye Service, Blackpool, UK

Correspondence: R Malhotra, Department of Ophthalmology, CorneoPlastic Unit, Queen Victoria Hospital NHS Foundation Trust, Holtye Road, East Grinstead, West Sussex RH19 3DZ, UK

Tel: + $44(0) 1342414549$;

Fax: + 44 (0)1342 414106

E-mail: Raman.malhotra@ qvh.nhs.uk

Received: 11 May 2011 Accepted: 27 May 2011 Published online: 12 August 2011

\begin{abstract}
Purpose To evaluate the use and safety of temporary, cosmetic, painted prostheses as an alternative to clear conformers immediately post removal of eye or socket surgery in children and adults.

Patients and methods A 2-year, two-centre, retrospective audit of 54 patients undergoing enucleation, evisceration, secondary implant or socket reconstruction receiving a temporary painted prosthesis as an alternative to clear conformers immediately post surgery. Patient questionnaire and review of clinical notes was conducted for all patients.
\end{abstract}

Results We reviewed 54 consecutive patients (22 females, 32 males, mean age 18.6 years, range 6 days to 82 years) who received cosmetic painted prostheses from January 2009 to December 2010. Procedures included: evisceration with primary implant (5), enucleation with primary implant (37), secondary implant (9), dermis fat graft (2) and fornix reconstruction with buccal graft (1). Complications included: pyogenic granuloma (2), mucous discharge (2) and exposure (2). All adults found their prosthesis comfortable, and no problems were reported by the parents of the children included in this study. In all, 46 patients ( $88 \%$ of respondents) returned to normal activities within 2 weeks. The temporary prosthesis fell out at least once in $25 \%$ of cases. In all, $90 \%$ of respondents expressed a definite preference towards the painted shell over a clear shell.

Conclusion Temporary cosmetic painted prostheses placed immediately after removal of the eye or socket surgery are well tolerated and preferred to standard clear shells. Painted
I Avisar', JH Norris', S Quinn², D Allan², M McCalla ${ }^{3}$, D Dugdale ${ }^{4}$, M Parulekar ${ }^{3}$ and R Malhotra

prostheses, matched to the contralateral iris are a valuable option in anophthalmic surgery as an alternative to traditional clear conformers. Eye (2011) 25, 1418-1422; doi:10.1038/eye.2011.179; published online 12 August 2011

Keywords: anophthalmia; prosthesis; acrylic; painted

\section{Introduction}

The loss of an eye is a distressful event for both patient and family with impact on an individual's social and professional life. Of particular concern to patients (and parents where the patient is a child) is the cosmetic appearance in the immediate postoperative period. Currently, the use of a transparent conformer or shell post surgery is considered standard practise. The primary function of a conformer is to protect the wound and maintain forniceal depth. The latter is especially important when considering cosmetic rehabilitation with maintenance of socket volume and prevention of contraction. ${ }^{1}$ It is recognised that a permanent prosthesis is more likely to be well tolerated when fitted at approximately 6 weeks post surgery. ${ }^{2}$

The most commonly used conformers are transparent, the advantage being that the wound can be seen easily by the clinician with minimal manipulation. This transparency, however, can cause embarrassment; some patients consider visualisation of the underlying socket unsightly added to which the conformer does not resemble the contralateral eye. In certain cases, the patient may cover or patch 
the eye. In children, the commonest indication for enucleation is advanced intraocular retinoblastoma, and surgery is usually performed within a few days of diagnosis. In such cases, the distress caused by the diagnosis is compounded by the inadequate time available for the family to prepare for the post-operative appearance, which may also cause considerable upset to siblings.

Custom-made acrylic resin prostheses manufactured at the end of the surgery have been described, as well as the use of a pre-made prosthesis. ${ }^{5,7}$ In some cases, further modification of the prosthesis may be required. Early placement of similar temporary prostheses (5-12 days following surgery) have also been previously suggested. ${ }^{8}$

The purpose of this study was to evaluate the safety and value of temporary, cosmetic, painted prostheses as an alternative to clear conformers immediately post removal of eye or socket surgery in children and adults.

\section{Patients and methods}

We performed a retrospective audit of consecutive cases performed over the last 2 years. All cases were performed at either Queen Victoria Hospital (QVH) or the Birmingham Children Hospital (BCH). All patients (or parents where the patient was a child) completed a structured interview questionnaire in conjunction with a review of their medical notes.

Patients were included in the audit if they had undergone: (i) an enucleation or an evisceration with or without primary or secondary orbital implant, (ii) an orbital implant exchange or (iii) a socket reconstruction and in addition, received a stock, painted, temporary prosthesis.

\section{Manufacturing process of the painted prosthesis}

The prosthetic painted conformers are manufactured as stock prostheses by the local maxillofacial laboratory at QVH or by 'Orbital Prosthetic Supplies' (Maidenbower, Crawley, UK) for patients at $\mathrm{BCH}$. The conformers are manufactured from lightweight acrylic resins. Iris pupil units are firstly painted using dry ground artist pigments suspended in liquefied acrylic resin. This unit is dried thoroughly and sealed to a corneal unit using cyanoacrylate adhesive. The scleral shade acrylic polymethylmethacrylate (PMMA) resin is then mixed and packed into the conformer unit mould and the two halves of the flask placed together under a hydraulic press. The mould is then clamped and placed into the paco-bath polymerisation unit. When the acrylic resin has polymerised, the ocular conformer units are removed from the mould and trimmed until the iris pupil unit is visible. Pigmentation of the sclera is applied to give the characteristic staining and veining found normally in the natural eye. A coating of liquid PMMA resin is applied to the conformer unit to facilitate adhesion of the veining to the conformer unit, which is then set aside to dry. A separating medium is applied to the entire mould and the conformer unit is positioned in the mould again. A clear polythene sheet is placed over the unit over which the clear PMMA is packed in to the mould before being re-pressed to remove the excess material. The mould/ flask unit is positioned under a hydraulic press for a further $2 \mathrm{~min}$ before being placed into the paco-bath. Once the cycle is completed, the clear acrylic is trimmed to the desired shape, smoothed on a lathe using a bristle brush and pumice and then polished using a polishing mop. A 2-mm fenestration is drilled through the conformer unit to allow egress of postoperative fluid from the socket and through which topical medication can be administered (Figure 1). The conformer unit is then sterilised and ready to use. A set of eight prosthetic shells in various sizes (large/small), iris colours (brown/ green/blue) and orientations (right/left) are available in the operating theatre.

\section{Surgical procedure}

As previously described ${ }^{3}$ before surgery, the role of the conformer and the inherent limitations with regards to matching the exact the size and colour to the contralateral eye was explained to the patient and/or the family. Surgery was carried out in a routine manner. Following closure of the conjunctiva, the socket and fornices were assessed. Either a large of small-sized temporary prosthesis was selected to achieve eyelid closure or lagophthalmos of no more than $2 \mathrm{~mm}$.

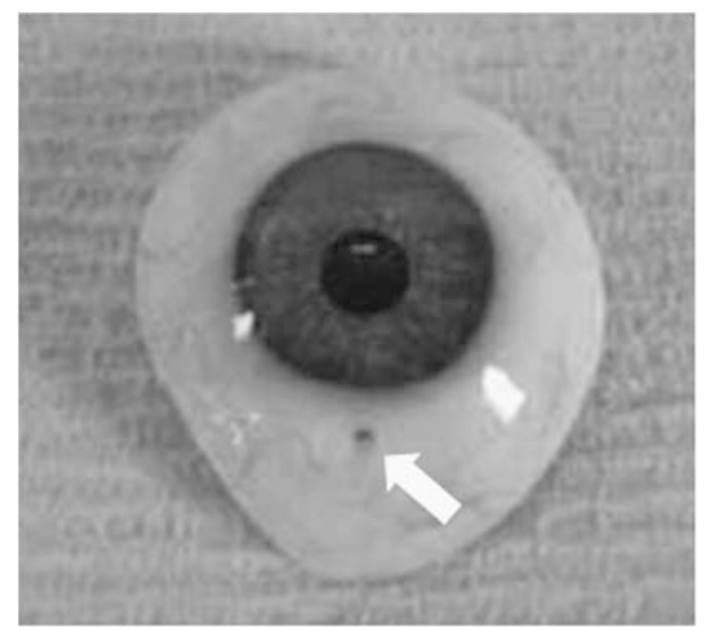

Figure 1 An example of a fenestrated (arrow) ocular acrylic stock painted prosthesis. 
Care was also taken to choose a large enough prosthesis that will optimise retention. The colour of the temporary prosthesis was then selected based on the colour of the contralateral eye. Postoperatively chloramphenicol ointment was applied to the socket. A Jelonet gauze (Smith \& Nephew Healthcare, Hull, UK) dressing along with a double eye pad was placed over the closed eye overnight.

The outcome measures included: (i) prosthesis related complications as reported from a clinical notes review,

(ii) cost of manufacture and finally (iii) patient reported acceptance of the cosmetic prostheses using a questionnaire.

At a minimum of 2 months, post-surgery patients or parents were asked a series of questions by telephone or within the clinic. They were asked to comment on the comfort of the temporary prosthesis, postoperative lifestyle changes and if a patch was worn after surgery. In addition, patients were asked to compare the temporary prosthesis with their final artificial eye specifically regarding iris colour match, degree of movement and comfort. Finally, in an attempt to compare the painted prosthesis and a standard clear shell, patients were asked for their overall cosmetic preference, and how they would have felt if they had been given a clear shell revealing their pink conjunctival socket when the eyelids were open. Complications including orbital haemorrhage, orbital infection, wound related complications and subsequent prosthetic fittings problems were recorded. The costs and manufacture procedure were also evaluated.

\section{Results}

In all, 54 consecutive patients ( 22 females, 32 males, mean age 18.6 years, range 6 days -82 years) were included in the study. In all, there were 36 paediatric patients aged $<10$ years of age who were included in the study. The remaining patients (18) were all adults over 27 years of age. All patients received cosmetic painted prostheses between January 2009 and December 2010. The surgical procedures performed included: evisceration with primary implant (5), enucleation with primary implant (37), secondary orbital implant (9), dermis fat graft (2) and fornix reconstruction with buccal graft (1). Indication for surgery included: retinoblastoma (34), Coats disease (2), a blind, painful eye (4), post-enucleation socket syndrome (2), orbital volume deficiency (9), exposure of orbital implant (2) and contracted socket (1).

In total, 14 adult patients had implants inserted, including hydroxyapetite (11) and acrylic (3) with various implant sizes: $22 \mathrm{~mm}$ (11), $20 \mathrm{~mm}$ (2) and $18 \mathrm{~mm}$ (1). All children had porous polyethylene implants (Medpor, Porex Surgical, Atlanta, GA, USA) in various sizes: $16 \mathrm{~mm}$ (1), $18 \mathrm{~mm}$ (7), $20 \mathrm{~mm}$ (15) and $22 \mathrm{~mm}$ (13).
Postoperative complications occurred in six patients $(11.1 \%)$, including pyogenic granuloma $(2,3.7 \%)$, mucous discharge treated by oral antibiotic $(2,3.7 \%)$ and implant exposure $(2,3.7 \%)$, of which one patient had early exposure (2 months post surgery) and one late exposure.

Overall, 52 patients or parents $(96.3 \%)$ completed the questionnaire. Of the two non-responders, one patient died and the other (both adults) declined due to difficulty understanding the questionnaire. All adult patients were comfortable with the printed prostheses, nine patients were comfortable 'most of the time' and seven patients were comfortable 'all the time'. In the adult group, $10(62.5 \%)$ returned back to work within 2 weeks, two patients (12.5\%) between 2 and 4 weeks and four patients $(25 \%)$ after $>4$ weeks. All children were reported to have gone back to normal activities within 2 weeks. Overall, $46(88 \%)$ returned to normal activities within 2 weeks.

In all, 11 adults $(68.7 \%)$ and all children (100\%) did not wear any patch/dark glasses after surgery. Two adults $(12.5 \%)$ used dark glasses and three adults $(18.7 \%)$ used a patch until a permanent prosthesis was available because of unsatisfactory appearance. Only three adults (18.7\%) reported changing their lifestyle due to surgery with less socialising and less going outdoors. In all, 13 patients (25\%) reported the temporary printed prosthesis fell out at least once and three (5.7\%) more than once, which required an exchange of the prosthesis. Patients were asked to compare their permanent prosthesis with their temporary printed shell placed immediately after surgery. In all, 26 patients (50\%) felt that the permanent prostheses had better movement and comfort compared with the temporary stock prosthesis. The remaining patients felt that same degree of comfort and movement was present in both prostheses. All patients felt colour match was better with their permanent prosthesis.

In all, 12 adult patients (75\%) and 35 of 36 children (97\%) would have preferred using the printed shell over a clear shell. Overall, $90 \%$ of respondents expressed a definite preference towards the painted shell over a clear shell (Figure 2). Four adults and one parent were indifferent and commented that as long as no complete match, the use of the printed prosthesis is of no benefit. Four parents suggested omitting the holes in the prosthesis to improve cosmesis (Figure 1).

For the adult patients, the permanent prosthesis was made locally by the prosthetists at QVH in eight patients (50\%), by the National Artificial Eye Service in six patients $(37 \%)$ and privately in two patients $(11 \%)$. In most paediatric cases, the permanent prosthesis was made mainly by the National Artificial Eye Service, although five patients had their prosthesis manufactured in either Scotland or Wales. The mean time period for 

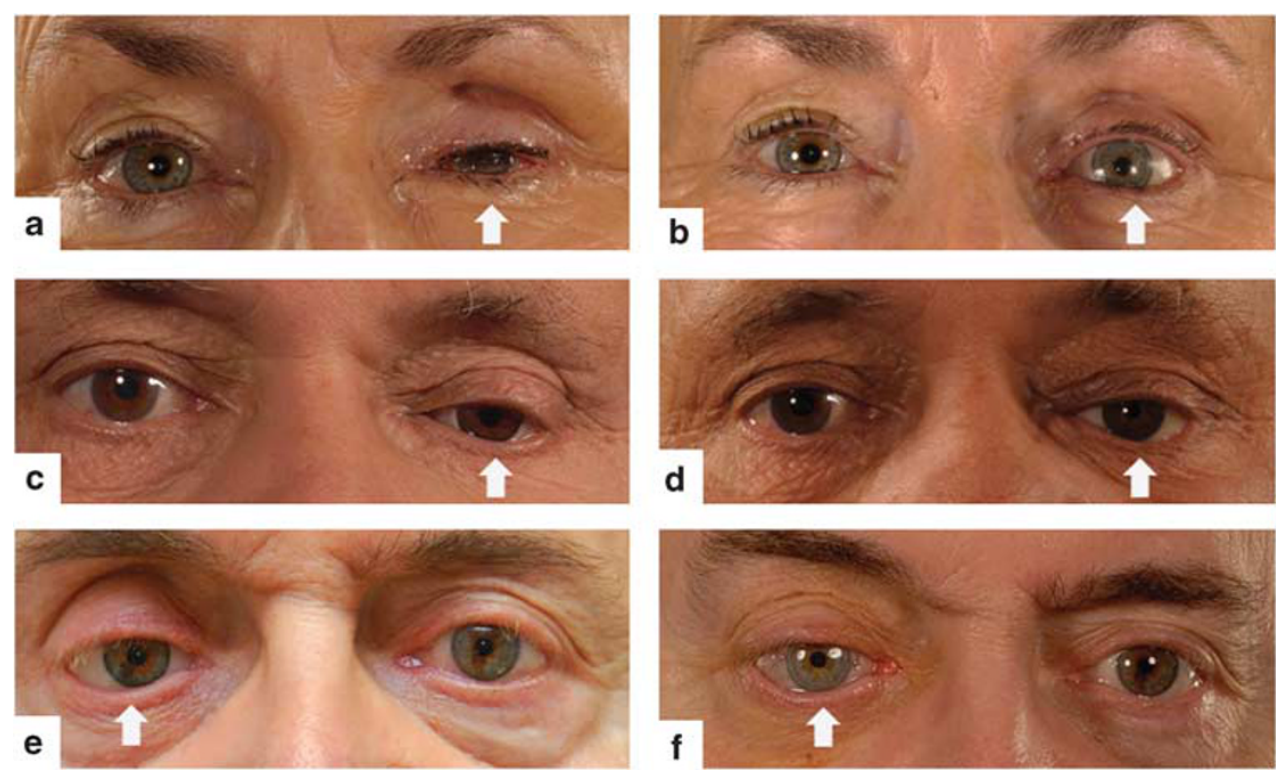

Figure 2 Patient pre- (a) and early-post (b) left evisceration with primary implant and temporary painted prosthesis. Patient pre- (c) and early-post (d) left secondary orbital implant exchange for a volume deficient socket. Patient pre- (e) and early-post (f) right secondary orbital implant exchange again for volume deficiency. This last case (f) illustrates the occasional difficulty in attaining an exact match of iris colour in some patients. The result is still aesthetically preferable when compared to a transparent conformer.

fitting of a permanent prosthesis was 6 months (range 4 weeks and 12 months) for all patients.

The costing (including labour and materials) for an ocular stock conformer is about four to five times more than the costing of the commercially manufactured clear shell conformers ( $£ 215$ compared with $£ 50$ ). However, the stock conformers also serve as a temporary ocular prosthesis, which is also required for all patients who have commercially manufactured clear shell conformers. Therefore, the use of the ocular stock prosthesis overall reduces postoperative costs.

\section{Discussion}

We found that a stock ocular prosthesis placed immediately after orbital implant surgery is tolerated well and is preferred by patients when compared with a standard clear shell. All patients were comfortable with the prosthetic shell and $90 \%$ of patients were pleased with the cosmetic benefit afforded by early introduction of the stock prosthesis. Of note, $90 \%$ of patients did not feel the need to wear a patch or dark glasses after surgery and $88 \%$ returned to work or regular activities within 2 weeks. However, the temporary printed prosthesis fell out at least once in $25 \%$ of patients.

Boissoneau, in 1849, was the first ocularist to produce stock glass eyes, which were popularised in Europe and America. ${ }^{6}$ Glass was the preferred prosthetic material at the time. However, owing to difficulty in modelling and its fragile nature, glass is seldom used today. The modern ocular prosthesis should be inert and easily mouldable and PMMA is currently the material of choice. ${ }^{6}$

Using pre-stocked painted prostheses has been previously described. Teicher et $a l^{7}$ described a technique for the use of prefabricated ocular prostheses immediately after the surgery. They subsequently modified this to produce the permanent prosthesis rather than using the standard technique. The main disadvantage to the use of these prefabricated eyes as a permanent prosthesis is the inability to match iris colour and size. Anecdotally, patients often comment that it is the actual iris colour that is the most important consideration in a permanent prosthetic eye.

Chin $e t \mathrm{al}^{8}$ found that the use of a prosthesis 5 to12 days post surgery had no effect on wound closure, final prosthetic fittings or movement of the permanent prosthesis. All patients in their series were pleased with the cosmetic benefit afforded by early introduction of the prosthesis and preferred their appearance to that of their eye with the conformer. ${ }^{8}$ This series differed from ours in terms of the timing; we place the artificial eye at the time surgery. We would argue that this confers certain advantages: first, the artificial eye can be placed while the patient is under general anaesthetic, which reduces pain and allows the patient improved appearance on waking. Immediate placement also permits a larger prosthesis to maintain the socket and tamponade the wound. 
Sykes et $a l^{5}$ described a method of fabricating acrylic resin, ocular, custom-made conformers, which can be used as an interim measure and can serve as an initial treatment option. They actually took a mould of the socket at the end of the surgery to produce a custommade conformer. They argued that these modified custom-made conformers can also be used to enlarge small or contracted sockets, stimulate eyelid movement, aid hygiene, help the clinician develop the final shape for the definitive prosthesis and reduce the post insertion adjustments needed. Such a process increases both the number of clinical visits and the production costs.

Vincent et $a l^{4}$ reported outcomes using printed prosthetic conformers placed at the end of the surgery in children. They report positive acceptance by families to a prosthetic conformer in this paediatric population thus improving rehabilitation of the family and anophthalmic child. However, no specific outcomes in terms of population size, quality of life outcomes and complications were provided.

Patel et $a l^{9}$ reported the designed interim printed prosthetic conformers to help patients overcome some of the problems of a clear conformer, commonly used. They designed two sets of kits. Each kit consists of six sizes in six different iris colours. One set made from a cream scleral colour and the other made for blue-white scleral colour. The centre of the conformer had a hole for drainage. In their study, they placed a clear conformer immediately after the surgery and after 5-7 days they placed the painted conformers. They concluded that the painted conformers can also be used immediately after surgery and suggested that an appointment with an ocularist before surgery would be beneficial, allowing a matched conformer to be used after the procedure.

The disadvantage of a stock prosthesis is the occasional reduction in apposition between the conjunctiva and the posterior prosthetic surface, which can lead to unequal weight distribution and irritation to the socket. This may present in the form of chronic discharge and laxity of the lower eyelid in the patient in the long term. In comparison, a custom moulded prosthesis allows even distribution of prosthesis volume and weight in the socket giving better cosmetic and less discomfort to the patient. However, the principal advantage of the stock painted prosthesis is the superior cosmetics with improved psychosocial well-being.

Orbital haemorrhage, infection and immune deficiency are relative contraindications for early prosthetic insertion. ${ }^{8}$ In cases of wound dehiscence, early placement of an ocular prosthesis may cause the stress on the wound and will prevent direct view of the socket (as possible with a transparent conformer); however, no patients in this series had such a complication.
We believe the use of a painted conformer placed immediately after orbital implant surgery is both well tolerated and preferred when compared to a standard clear shell. We do acknowledge the lack of a prospective comparative group using only clear conformers.

Although a relatively large prospective quality-of-life study may be required for statistical proof of benefit; in this series, 47 patients (90\%) preferred the early cosmetic rehabilitation associated with placement of an ocular prosthesis. We recommend where possible the routine use of stock painted conformers during anophthalmic surgery requiring the use of a temporary prosthesis.

\section{Summary}

What was known before

- The most commonly used conformers are transparent, the advantage being that the wound can be seen easily by the clinician with minimal manipulation. This transparency however can cause embarrassment; some patients consider visualization of the underlying socket unsightly added to which the conformer does not resemble the contralateral eye.

What this study adds

- We believe the use of a painted conformer placed immediately after orbital implant surgery is both well tolerated and preferred when compared with a standard clear shell. In this series, 47 patients (90\%) preferred the early cosmetic rehabilitation associated with placement of an ocular printed prosthesis.

\section{Conflict of interest}

The authors declare no conflict of interest.

\section{References}

1 Custer P, Cook B. The team approach to the anophthalmic patient. Adv Ophthalmic Plast Reconstr Surg 1990; 8: 55-57.

2 Collin JRO. A Manual of Systematic Eyelid Surgery. Churchill Livingstone: London, 1989, p 124.

3 Brett J. Creating a treatment plan for enucleation or evisceration surgery. J Ophthalmic Prosthetics 2000; 5: 45-48.

4 Vincent AL, Webb MCF, Gallie BL, Héon E. Prosthetic conformers: a step towards improved rehabilitation of enucleated children. Clin Exp Ophthalmol 2002; 30: 58-59.

5 Sykes LM, Essop AR, Veres EM. Use of custom-made conformers in the treatment of ocular defects. J Prosthet Dent 1999; 82(3): 362-365.

6 Kuldeep Raizada, Deepa Rani. Ocular prosthesis. Cont Lens Anterior Eye 2007; 30(3): 152-162.

7 Taicher S, Steinberg HM, Tubiana I, Sela M. Modified stock-eye ocular prosthesis. J Prosthet Dent 1985; 54(1): 95-98.

8 Chin K, Margolin CB, Finger PT. Early ocular prosthesis insertion improves quality of life after enucleation. Optometry 2006; 77(2): 71-75.

9 Patel BC, Sapp NA, Collin JR. Cosmetic conformers. Ophthalmic Surg Lasers 1997; 28(2): 171-173. 